\title{
Nodular Fasciitis
}

\section{Characteristic Imaging Features on Sonography and Magnetic Resonance Imaging}

\author{
Anthony Khuu, MD, Corrie M. Yablon, MD, Jon A. Jacobson, MD, Alero Inyang, MD, \\ David R. Lucas, MD, J. Sybil Biermann, MD
}

Received June 10, 2013, from the Departments of Radiology (A.K., C.M.Y., J.A.J.), Pathology (A.I., D.R.L.), and Orthopedic Surgery (J.S.B.), University of Michigan, Ann Arbor, Michigan USA. Revision requested July 1, 2013. Revised manuscript accepted for publication August 5, 2013.

Address correspondence to Corrie $M$. Yablon, MD, Department of Radiology, University of Michigan, Taubman Center, Room 2910Q, 1500 E Medical Center Dr, Ann Arbor, MI 48109-5326 USA.

E-mail:cyablon@umich.edu

Abbreviations

MRI, magnetic resonance imaging
Objectives - The purpose of this study was to evaluate the imaging features of nodular fasciitis on sonography and magnetic resonance imaging (MRI).

Methods-A retrospective search of the radiologic and pathologic databases over the past 10 years for the diagnosis of nodular fasciitis was performed. Sonographic and MRI features were described. Pathologic specimens were reviewed.

Results - Six pathologically confirmed cases of nodular fasciitis were found. The mean patient age was 19.5 years (range, $8-33$ years); 3 patients were male and 3 were female. Four patients had sonography only; 1 patient had both sonography and MRI; and 1 patient had MRI only. Three masses were located in the subcutaneous tissue adjacent to fascia; 2 were at the subcutaneous/muscular border; and 1 was intramuscular; however, all were in contact with fascia and showed a fascial tail on sonography and MRI. On sonography and MRI, masses were oval with poorly defined lobulated borders, averaging $2.6 \mathrm{~cm}$ (range, 1.8-3.5 cm). On sonography, all masses were hypoechoic. On MRI, the masses were isointense to muscle on T1-weighted sequences, hyperintense to muscle on fluid-sensitive sequences, and enhanced avidly but heterogeneously. The masses were surrounded by fat.

Conclusions - When a rapidly growing oval mass in contiguity with a fascial plane is recognized, the diagnosis of nodular fasciitis should be entertained. More importantly, the possibility of an inaccurate diagnosis by core biopsy exists, which may warrant gross resection.

Key Words - musculoskeletal ultrasound; nodular fasciitis; nodular fasciitis diagnosis; nodular fasciitis imaging; sonography

$\mathrm{N}$ odular fasciitis, also known as infiltrative or pseudosarcomatous fasciitis, is a benign soft tissue tumor of fibroblastic/myofibroblastic differentiation that was first described in 1955 by Konwaler et al. ${ }^{1}$ The lesion often comes to clinical attention when a patient presents with a rapidly growing, occasionally painful, palpable soft tissue mass. ${ }^{2,3}$ Although benign and self-limited, nodular fasciitis is a clonal neoplastic proliferation characterized by recurrent MYH9-USP6 (myosin heavy chain 9-ubiquitin-specific peptidase 6) gene fusion. ${ }^{4}$

Despite the fact that in a large retrospective study nodular fasciitis was found to represent $11.3 \%$ of benign soft tissue tumors, ${ }^{5}$ relatively few descriptions of nodular fasciitis have appeared in the radiologic literature. Most of the reports have been published in 
oncologic, pathologic, and dermatologic literature. The imaging appearance has been described as nonspecific and mimicking other sarcomatous lesions. On sonography, occasional case reports have described the lesion as oval or lobulated and hypoechoic to muscle or of mixed echogenicity. ${ }^{2,6}$ On magnetic resonance imaging (MRI), nodular fasciitis has been described as inhomogeneous, hypointense to muscle on T1-weighted images, and hyperintense to fat on $\mathrm{T} 2$-weighted images, with variable enhancement. $2,5,7,8$

Given the relatively variable reported imaging features of nodular fasciitis, we proposed in our study to characterize nodular fasciitis both on sonography and MRI with the intention of identifying imaging features that may help prospectively diagnose this benign lesion.

\section{Materials and Methods}

\section{Patients and Clinical Data}

Institutional review board approval was obtained for this Health Insurance Portability and Accountability Actcompliant study. We retrospectively searched the radiologic and pathologic databases using the search term "nodular fasciitis" between January 2002 and August 2012. Inclusion criteria were patients with the diagnosis of nodular fasciitis on imaging and patients who received the diagnosis based on percutaneous core biopsy or gross resection. Exclusion criteria were patients who lacked imaging on our picture archiving and communication system and who lacked a final diagnosis of nodular fasciitis on gross specimens. If patients had outside hospital images available for review, then these patients were included. Patient demographics, clinical records, and radiologic reports were reviewed.

\section{Imaging}

Sonograms were acquired with high-frequency linear array $(12-17 \mathrm{MHz})$ transducers on clinical ultrasound machines (LOGIQ 9; GE Healthcare, Wauwatosa, WI; and HDI 5000 and iU22; Philips Healthcare, Bothell, WA) as part of routine patient care. Magnetic resonance images were acquired on a clinical 1.5-T system (Signa Excite; GE Healthcare). The MRI tumor protocol parameters varied across platforms and institutions but consisted of the following sequences: axial proton density T1-weighted and fat-saturated T2-weighted images, coronal T1-weighted and short-tau inversion recovery images, sagittal fatsaturated T2-weighted turbo spin echo images, and axial fat-saturated spoiled gradient precontrast and postcontrast images.

\section{Image Analysis}

Two musculoskeletal radiologists with 8 and 18 years of experience retrospectively reviewed all sonograms and MR images on a digital workstation in consensus. The location of the lesion was categorized as subcutaneous, subfascial, or intramuscular. The lesions were characterized by sonography according to their echogenicity relative to the adjacent muscle (anechoic, hypoechoic, isoechoic, or hyperechoic), uniformity (homogeneous or heterogeneous), margins (well or poorly defined), shape (round, oval, or other), hyperemia, shadowing, calcification, and increased through-transmission. The presence of a fascial tail and the direct contiguity of the lesion with the fascia were documented.

Magnetic resonance imaging features of the lesions were characterized according to the location, size, signal intensity, and margin. The border of the lesion was classified as well or poorly defined, and the shape was categorized as round, oval, or other. Signal intensity on T1-weighted and fluid-sensitive MR images was described as low, equal, or high and as either homogeneous or heterogeneous. The degree of enhancement was subjectively assessed as being none, moderate, or avid. The lesion was also evaluated for the presence of a fascial tail and for the presence of fat surrounding the mass.

\section{Histologic Analysis}

Two pathologists with 5 and 25 years of experience reviewed the cases with the final diagnosis of nodular fasciitis. The lesion shape and margins were described. Lesions were assessed for cystic changes, myxoid changes, cellularity, the presence of a fascial attachment, and infiltration of adjacent muscle.

\section{Results}

Nineteen patients with either an imaging or a pathologic diagnosis of nodular fasciitis were identified. Seven patients were excluded who had an initial core biopsy diagnosis of nodular fasciitis but then subsequently received a different final pathologic diagnosis on the resection specimen resulting in a change of the final diagnosis to myxoma or fibromatosis. Two patients with a final diagnosis of nodular fasciitis were excluded because they lacked correlative imaging for review. Four patients had a preliminary diagnosis of nodular fasciitis but lacked a final pathologic diagnosis based on the resection specimen.

The 6 remaining patients formed the final study population. They had both preprocedural imaging at our institution and a final diagnosis of nodular fasciitis on gross 
specimens. In 2 of 6 cases, the preliminary core biopsy showed nodular fasciitis, and the final pathologic diagnosis in the resection specimen remained nodular fasciitis. In 1 of 6 cases, an outside hospital diagnosed fibromatosis on core biopsy, but the final gross pathologic diagnosis was nodular fasciitis. In 3 of 6 cases, no preliminary core biopsies were performed.

The study group (mean age, 19.5 years; range, $8-33$ years) included 3 male patients (mean age, 17.3 years; range, $8-33$ years) and 3 female patients (mean age, 21.7 years; range, $18-28$ years). Of these, 4 lesions were imaged by sonography only; 1 lesion was imaged by MRI only; and 1 was imaged by both MRI and sonography.

Five of the 6 patients presented to their physicians with a history of an enlarging palpable mass. Three of these 5 patients noted pain at the site of the mass. One patient's mass was found incidentally on pelvic computed tomography performed to assess pelvic disease. The mean time to presentation of the 5 patients was 4 months (range, 2-9 months). None of the patients reported a history of trauma.
The morphologic features of the 6 cases of nodular fasciitis are summarized in Table 1. Four lesions were located in the lower extremity; 1 was located in the hand; and 1 was located in the upper posterior chest wall. Two lesions were located in the subcutaneous tissues; 3 were located in the fascial plane between the subcutaneous layer and the muscle (subfascial); and 1 was located within the vastus medialis muscle (intramuscular type). No lesions invaded osseous structures.

All lesions were small; the sizes ranged from 1.8 to 3.5 $\mathrm{cm}$ in greatest diameter, with a mean diameter of $2.6 \mathrm{~cm}$. All lesions were oval, and the margins were poorly defined or microlobulated on both MRI and sonography. All lesions contacted the fascia, and a fascial tail was visible on both MRI and sonography (Figures 1 and 2).

Imaging findings are summarized in Table 2 . The 5 lesions imaged with sonography all showed mixed echogenicity but were predominantly hypoechoic (Figure 3A). Three of the 5 lesions showed moderately increased color Doppler flow (Figure 3B). All but the smallest lesion showed increased through-transmission (Figure 4). None of the 5 lesions showed shadowing or internal calcifications.

Table 1. Morphologic Features of Nodular Fasciitis Lesions

\begin{tabular}{lllccc}
\hline Patient & Age, $\mathbf{y} /$ Sex & \multicolumn{1}{c}{ Location } & Size, $\mathbf{c m}$ & Shape/Margin & Abuts Fascia/Facial Tail \\
\hline 1 & 28/female & Subcutaneous fat, lower leg & 1.8 & Oval/poorly defined & Yes/yes \\
2 & 19/female & Subfascial, thigh & 3.5 & Oval/poorly defined & Yes/yes \\
3 & 8/male & Subfascial, hand & 3.5 & Oval/poorly defined & Yes/yes \\
4 & 33/male & Intramuscular/vastus medialis & 2.5 & Oval/poorly defined & Yes/yes \\
5 & 11/male & Subcutaneous fat/popliteal region & 2.2 & Oval/poorly defined & Yes/yes \\
6 & 18/female & Subfascial/periscapular & 2.0 & Oval/poorly defined & Yes/yes \\
\hline
\end{tabular}

Figure 1. Nodular fasciitis of the lower leg in a 28-year-old woman. A, Axial fat-saturated T2-weighted MRI shows an oval lobulated subcutaneous lesion (thick arrows) that is hyperintense to skeletal muscle, with extension along deep anterior fascia, consistent with a fascial tail (thin arrow). B, T1-weighted MRI shows that the lesion (arrow) is isointense to muscle with a T1 hyperintense rim, consistent with fat.

A

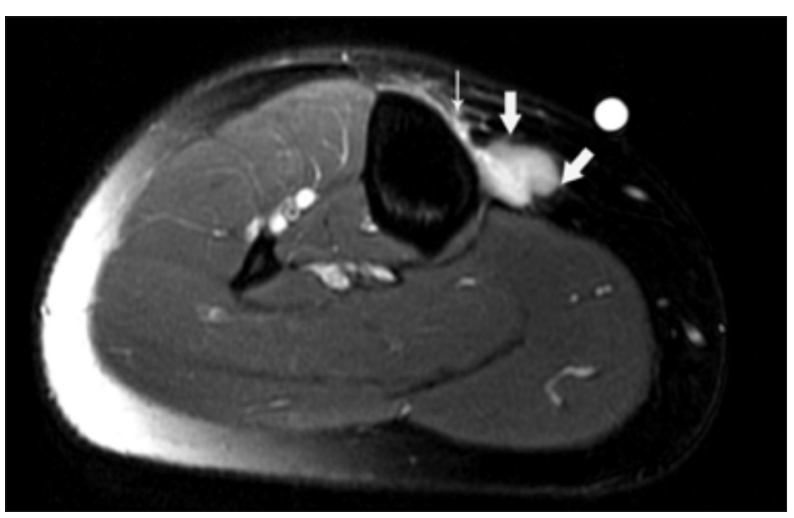

B

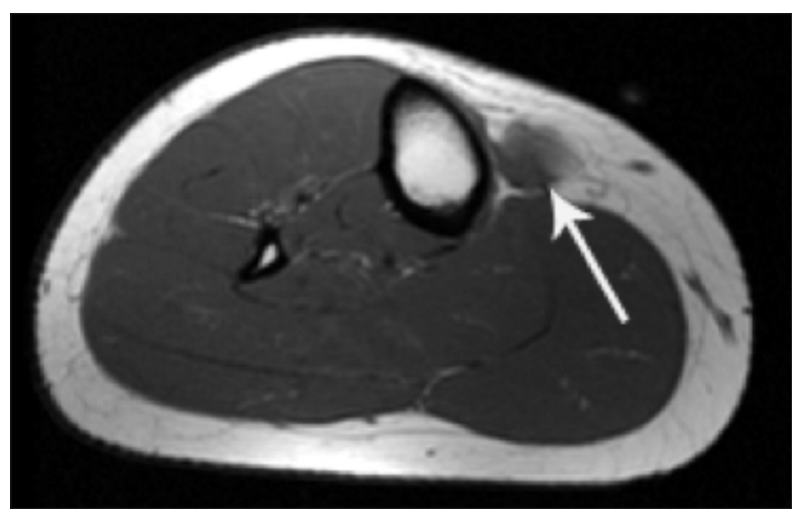


On MRI, 2 lesions (patients 1 and 2) showed homogeneous signal intensity that was isointense to normal muscle on T1-weighted images. Compared to muscle, both lesions were hyperintense on $\mathrm{T} 2$-weighted images. There was no surrounding hyperintensity on T2weighted images to suggest perilesional or soft tissue

Figure 2. Nodular fasciitis of the thigh in a 19-year-old woman. A, Sonography shows an intermuscular or subfascial hypoechoic mass occurring between the tensor fascia lata and gluteus medius muscle. The lesion is contiguous with and extends along the superficial border of the gluteus medius, consistent with a fascial tail (arrows). B-D, Magnetic resonance imaging shows a subfascial or an intermuscular oval lesion that is isointense to muscle on T1-weighted imaging ( $\mathbf{B}$, arrow), is hyperintense to muscle on fatsuppressed T2-weighted imaging ( $\mathbf{C}$, arrow), and shows avid enhancement on fat-suppressed T1-weighted postcontrastimaging (D, arrow) (continued).

A

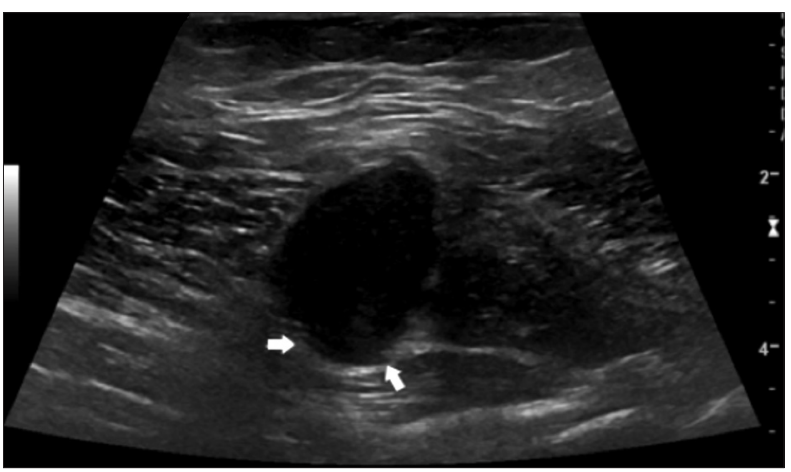

B

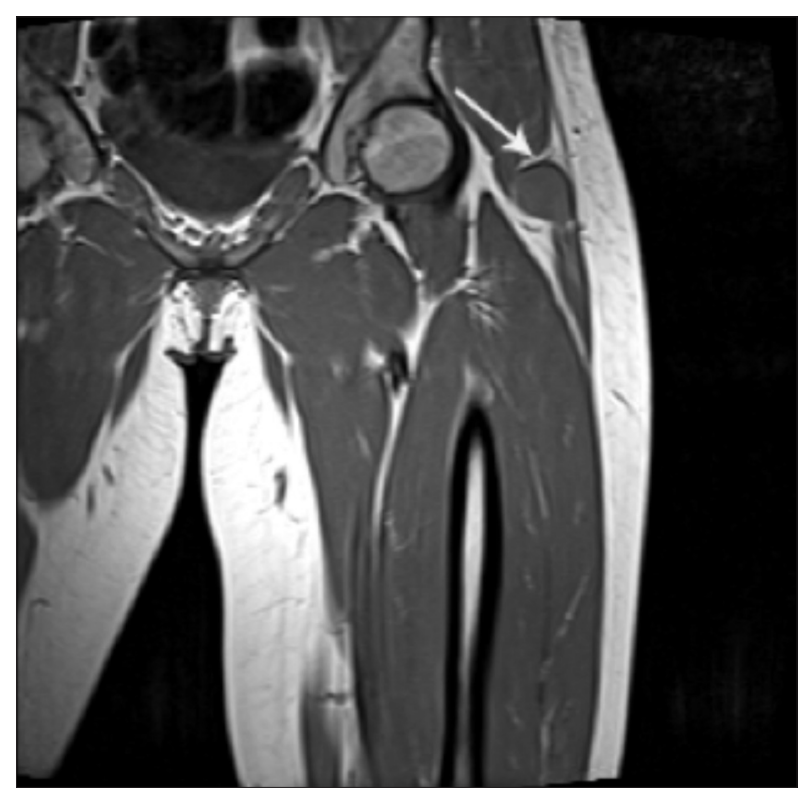

edema. In both MRI cases, a thin rim of T1 hyperintense signal consistent with fat was identified (Figures $1 \mathrm{~B}$ and $2 \mathrm{~B})$. Of the $2 \mathrm{MRI}$ cases, only 1 received contrast agent; after gadolinium contrast agent administration, this lesion showed avid, somewhat heterogeneous enhancement (Figure 2D).
C

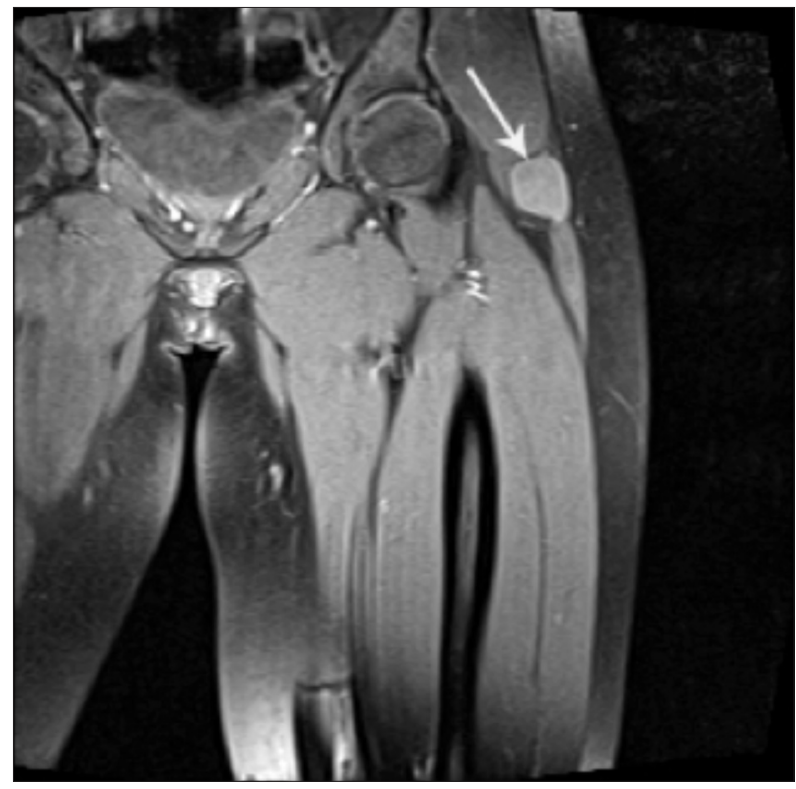

D

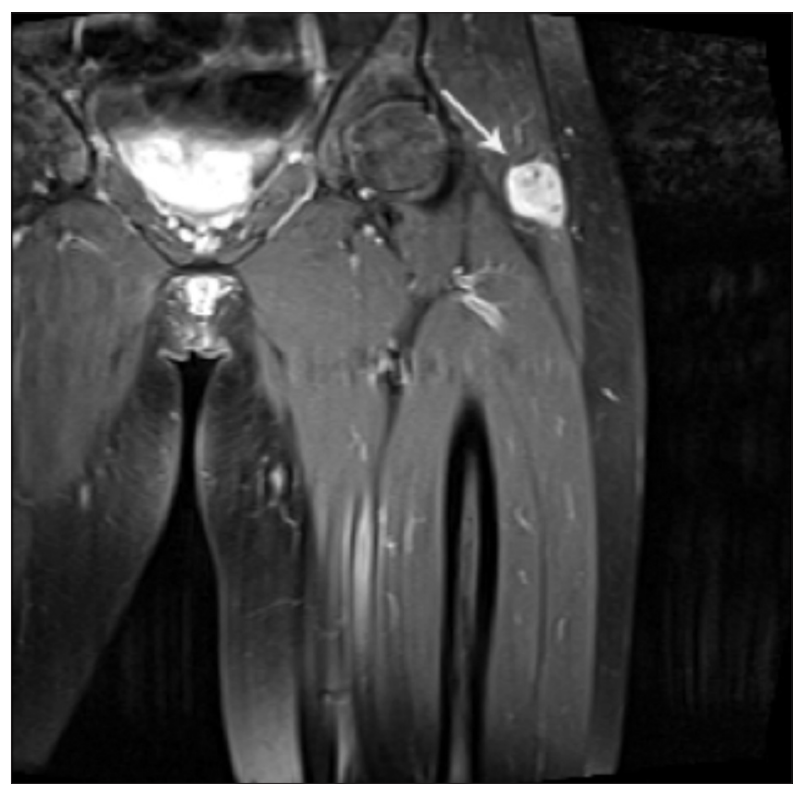


Figure 2. (continued) E, Microscopically, nodular fasciitis is characterized by plump fibroblasts/myofibroblasts arranged in short irregular bundles amid fibromyxoid stroma with a feathery appearance (arrows; hematoxylin-eosin, original magnification $\times 200$ ). F, Fascial attachment (arrows) was identified in 3 tumors, corresponding to fascial tails identified by imaging (hematoxylin-eosin, original magnification $\times 20$ ).

E

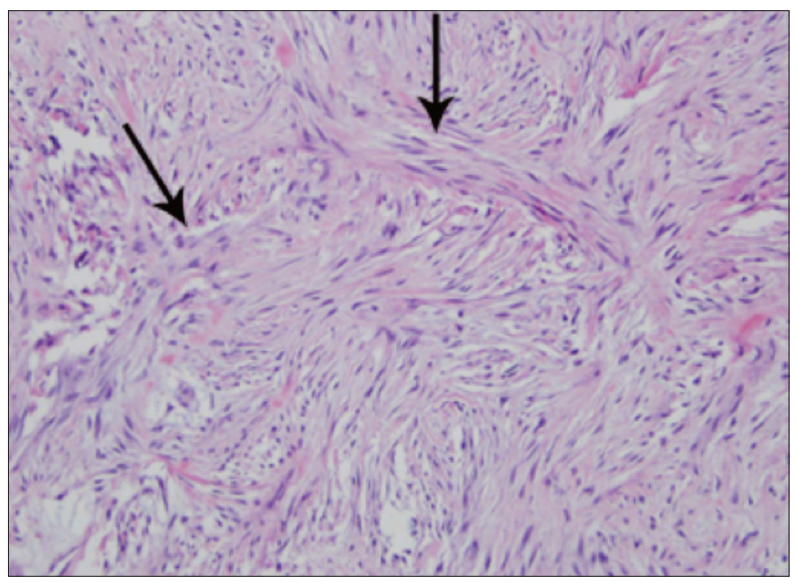

$\mathbf{F}$

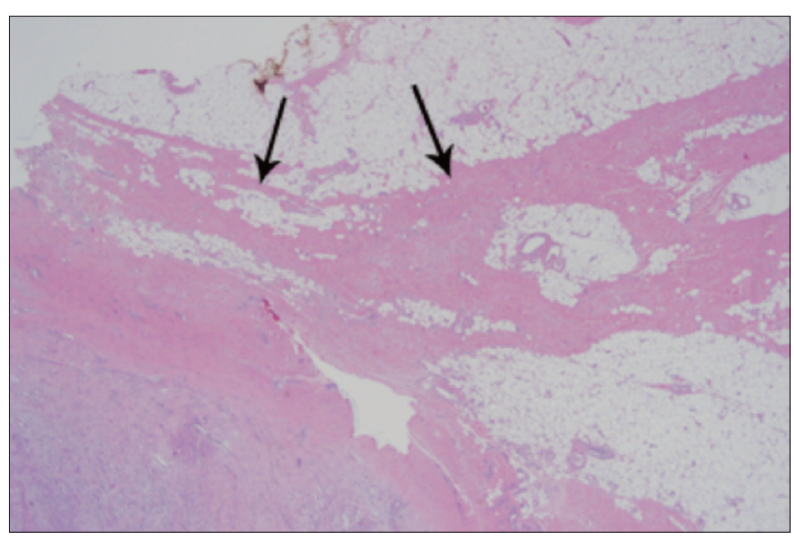

Slides from the surgical excisions specimens were reexamined by 2 pathologists in consensus, and the results are listed in Table 3. Patients $1-5$ had slides available for review; patient 6 had a diagnosis based on outside pathologic slides, and those slides could not be reobtained for review. The 5 tumors that were reviewed showed the common features of nodular fasciitis, consisting of fascicles of benign fibroblastic/myofibroblastic cells within a loosely textured fibromyxoid matrix (Figure 2E). Four of 5 lesions had an oval appearance, whereas 1 was round. Four had well-defined borders, and 1 had an irregular border. One lesion showed cystic changes. Three lesions were attached to fascia (Figure 2F), whereas 2 had no identifiable fascial attachment. Two lesions showed high cellularity; 2 showed mixed but predominantly high cellularity; and 1 showed low cellularity. Four of 5 lesions showed myxoid changes, and 1 did not. Two of 5 lesions were found to infiltrate muscle.

The radiologic reports of the cases with a final pathologic diagnosis of nodular fasciitis were reviewed to determine the prebiopsy prospective diagnosis. In 3 of the lesions, a differential diagnosis including a peripheral nerve sheath tumor, fibromatosis, a giant cell tumor, or lipoma was given. In 3 of the lesions, a differential diagnosis was not given, and the lesions were described as nonspecific, with an inability to exclude tumors.

\section{Discussion}

The imaging description of nodular fasciitis is limited in the literature, with variable sonographic and MRI findings. Konwaler et $\mathrm{al}^{1}$ originally described nodular fasciitis as pseudosarcomatous fibromatosis, a benign, self-limited reactive process composed of proliferating fibroblasts in a

Table 2. Imaging Features of Nodular Fasciitis

\begin{tabular}{|c|c|c|c|c|c|c|c|}
\hline \multirow[b]{2}{*}{ Patient } & \multirow[b]{2}{*}{$\begin{array}{c}\text { Age, y/ } \\
\text { Sex }\end{array}$} & \multicolumn{3}{|c|}{ MRI } & \multicolumn{3}{|c|}{ Sonography } \\
\hline & & $\begin{array}{l}\text { T1 Signal } \\
\text { Intensity }\end{array}$ & $\begin{array}{l}\text { T2 Signal } \\
\text { Intensity }\end{array}$ & Enhancement & Echogenicity & $\begin{array}{l}\text { Hyperemia/ } \\
\text { Through- } \\
\text { transmission }\end{array}$ & $\begin{array}{c}\text { Shadow/ } \\
\text { Calcification }\end{array}$ \\
\hline 1 & 28/female & $\begin{array}{l}\text { Homogeneous, } \\
\text { isointense }\end{array}$ & $\begin{array}{l}\text { Homogeneous, } \\
\text { hyperintense }\end{array}$ & No contrast & Not determined & Not determined & Not determined \\
\hline 2 & 19/female & $\begin{array}{l}\text { Homogeneous, } \\
\text { isointense }\end{array}$ & $\begin{array}{l}\text { Heterogeneous, } \\
\text { hyperintense }\end{array}$ & $\begin{array}{l}\text { Heterogeneous, } \\
\text { avid }\end{array}$ & $\begin{array}{l}\text { Homogeneous, } \\
\text { hypoechoic }\end{array}$ & Yes/yes & No/no \\
\hline 3 & $8 /$ male & Not determined & Not determined & Not determined & $\begin{array}{l}\text { Heterogeneous, } \\
\text { hypoechoic }\end{array}$ & No/yes & No/no \\
\hline 4 & 33/male & Not determined & Not determined & Not determined & $\begin{array}{l}\text { Heterogeneous, } \\
\text { hypoechoic }\end{array}$ & Yes/yes & $\mathrm{No} / \mathrm{no}$ \\
\hline 5 & 11/male & Not determined & Not determined & Not determined & $\begin{array}{l}\text { Heterogeneous, } \\
\text { hypoechoic }\end{array}$ & Yes/yes & $\mathrm{No} / \mathrm{no}$ \\
\hline 6 & 18/female & Not determined & Not determined & Not determined & $\begin{array}{l}\text { Homogeneous, } \\
\text { hypoechoic }\end{array}$ & $\mathrm{No} / \mathrm{no}$ & $\mathrm{No} / \mathrm{no}$ \\
\hline
\end{tabular}


myxoid stroma. Prior reports have described the lesion as commonly occurring in younger adults in the third to fourth decades, ${ }^{7-9}$ predominantly in the upper extremity. $3,7,8,10$ Although most cases of nodular fasciitis are centered in the fascia, they can also be dermal, subcutaneous, deep fascial, intermuscular, intramuscular, and intravascular. ${ }^{3,5,7,11-13}$ In the study by Bernstein and Lattes, ${ }^{3} 85 \%$ of patients with nodular fasciitis were younger than 50 years.

On gross dissection, nodular fasciitis appears as a $\tan$ oval well-circumscribed nodule without a definite capsule. ${ }^{1}$ The pathologic diagnosis is made by identifying immature plump fibroblasts similar in size, arranged in short irregular bundles and fascicles, with a "feathery" appearance, and abundant myxoid changes. Brisk mitotic activity is usually present, but without cellular atypia. Myxoid, cellular, and fibrous subtypes have been described. ${ }^{3,10}$ In some reports, the histologic subtype was thought to correlate with the age of the lesion. $3,7,10,14$

The aggressive clinical presentation and imaging appearance of nodular fasciitis will usually prompt a biopsy for definitive diagnosis. Several case reports have described spontaneous regression or a decreased size of the lesion after percutaneous biopsy. ${ }^{3,15}$ However, because of their rapid growth, these lesions are usually managed surgically and resected. There is a low incidence of recurrence. ${ }^{3}$
In our study, we found that our patient population, with a mean age of 19.5 years, was considerably younger than previously reported in the literature. This finding was likely due to a referral bias, in which older patients with masses are more likely to have biopsy and diagnosis in the community, whereas children with masses may be referred to a tertiary center, especially in the absence of previous trauma.

Although prior literature describes upper extremity predominance, in this study, nodular fasciitis occurred more commonly in the lower extremity, a finding that differs from other previously published data. This finding should raise the possibility of nodular fasciitis in the differential diagnosis of a rapidly growing palpable mass in the lower extremities.

The locations of the lesions were similar to those in previously published reports. All were contiguous with the fascia on imaging. There were no morphologic differences seen in the lesions based on the location, as previously described. In addition, there was no intra-osseous or intraarticular extension of the lesions, as described in other publications. ${ }^{11,16-18}$

The sizes of the lesions in our study were comparable to those in other studies, with a mean diameter of $2.6 \mathrm{~cm}$. The mean time from lesion discovery to presentation for

Figure 3. Nodular fasciitis of the thigh in a 33-year-old man. A, Sonography shows an intramuscular lesion located in the vastus medialis that has mixed echogenicity but is predominantly hypoechoic (arrow). The border is microlobulated. B, Color Doppler sonography shows increased color Doppler flow internally and at the periphery of the lesion.

A

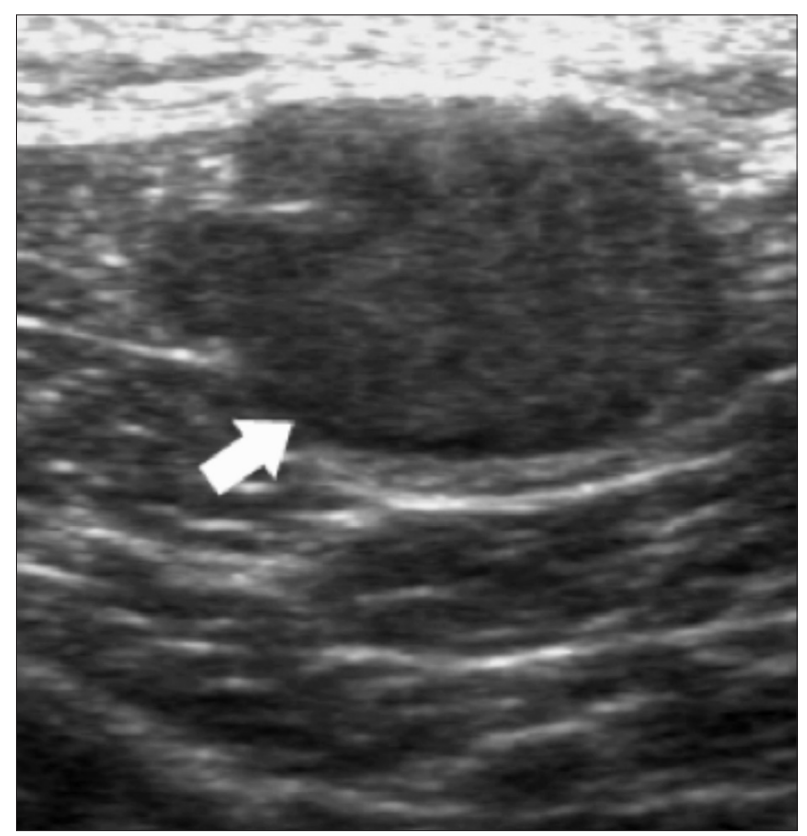

B

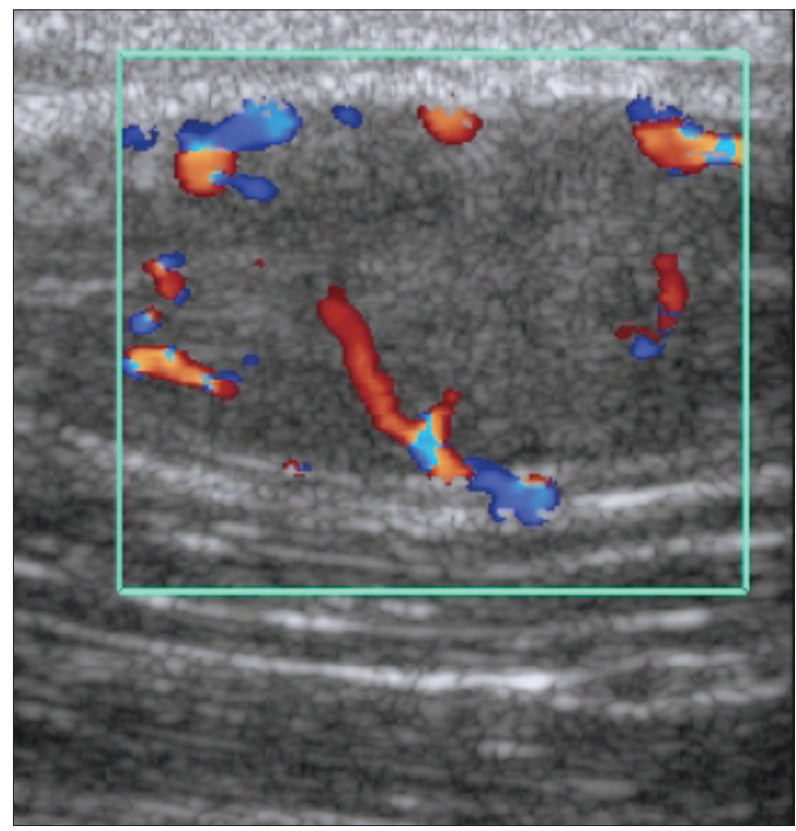




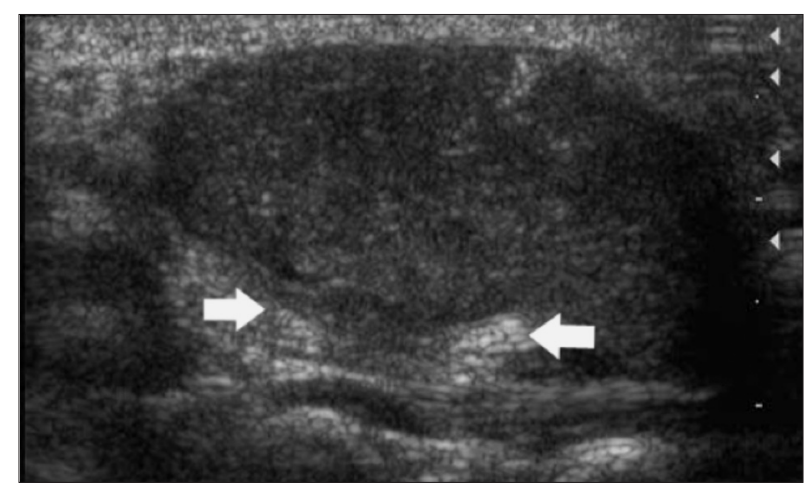

Figure 4. Nodular fasciitis of the hand in an 8-year-old boy. Sonography shows a hypoechoic mass with increased through-transmission (arrows).

treatment was 4 months (range, 2-9 months), which is similar to what has been previously published. In prior studies, a small subset of patients reported a history of trauma to the area in which nodular fasciitis was subsequently diagnosed. ${ }^{3}$ None of our patients reported a history of trauma. In 1 of the 6 cases (the periscapular lesion), a history of spontaneous diminishment in size was noted. Given the recent discovery of a genetic mutation associated with the clonal proliferation of nodular fasciitis, the previously described association between trauma and nodular fasciitis may be purely incidental.

The 5 lesions imaged with sonography all appeared predominantly hypoechoic with mixed echogenicity, concordant with other published sonographic case reports. ${ }^{2,6}$ All but the smallest lesion revealed increased posterior acoustic enhancement, or through-transmission, as described in a case report. ${ }^{2}$ None of the 5 lesions showed shadowing or internal calcification; however, internal echogenic foci have been described in one case report. ${ }^{2}$ Although "substantial color Doppler arterial flow" has been described, we only saw modest to scant color Doppler flow in 3 of 5 lesions. All lesions abutted the fascia and showed a fascial tail, a finding that, to our knowledge, has not been previ- ously described in the sonographic literature; it is important in that its presence may suggest nodular fasciitis as a potential diagnosis.

The 2 lesions imaged with MRI (patients 1 and 2) had similar imaging findings to those described in prior reports. ${ }^{7,8,11,14}$ However, we did not find perilesional hyperintensity on T2-weighted images to suggest soft tissue edema, a finding recently described. ${ }^{11}$ To our knowledge, the MRI finding of a thin rim of T1 hyperintense signal consistent with fat has not been previously described, and it may aid in making a diagnosis of nodular fasciitis.

On histologic analysis, there was variable cellularity, cystic changes, fascial attachment, and infiltration of muscle; however, most of the lesions ( 4 of 5) showed myxoid changes. Other studies have speculated that the histologic subtype of nodular fasciitis corresponds to the anatomic location or the age of the lesion. ${ }^{14} \mathrm{We}$ did not find this relationship to be the case in our patient population.

We found few pathologically proven nodular fasciitis lesions in our tertiary referral population, despite the reports in the literature that nodular fasciitis is relatively common. This finding may reflect a referral bias, in that these masses may be biopsied and treated in the community.

In the literature, reported differential diagnoses for nodular fasciitis on MRI include an extra-abdominal desmoid tumor, neurofibroma, fibrous histiocytoma, and soft tissue sarcoma. ${ }^{7}$ On sonography, reported differential diagnoses of nodular fasciitis include a peripheral nerve sheath tumor, fibromatosis, lymph nodes, and sarcoma. ${ }^{2}$ The diagnosis of a peripheral nerve sheath tumor should be considered when a mass occurs along the expected distribution of a nerve, and the diagnosis is made more certain when a nerve is seen entering and exiting the lesion. Although the MRI findings of the "split-fat sign" and the "target sign" have been described, ${ }^{19}$ MRI findings of peripheral nerve sheath tumors are variable; however, the finding of a fascial tail, as described in this study, may aid diagnosis of nodular fasciitis, as a peripheral nerve sheath tumor is not seen to have a fascial tail.

Table 3. Histologic Characterization of Nodular Fasciitis

\begin{tabular}{llllccccc}
\hline Patient & Age, $\mathbf{y} /$ Sex & Shape & Margin & $\begin{array}{c}\text { Cystic } \\
\text { Changes }\end{array}$ & $\begin{array}{c}\text { Attached } \\
\text { Fascia }\end{array}$ & Cellularity & $\begin{array}{c}\text { Myxoid } \\
\text { Changes }\end{array}$ & $\begin{array}{c}\text { Infiltrated } \\
\text { Muscle }\end{array}$ \\
\hline 1 & $28 /$ female & Round & Well defined & No & Yes & Intermediate & Yes & No \\
2 & 19/female & Oval & Well defined & No & Yes & High & Yes & No \\
3 & 8/male & Oval & Well defined & No & No & High & Yes & Yes \\
4 & $33 /$ male & Oval & Well defined & Yes & No & Low & Yes & No \\
5 & 11/male & Oval & Poorly defined & No & Yes & High & Yes & Yes \\
\hline
\end{tabular}


Deep and superficial fibromatosis may be confused with nodular fasciitis on MRI, as it has similar signal characteristics and enhancement patterns. Low-signal intensity fibrous bands, representing collagenous material, have been described, but this finding is not always seen. Deep fibromatosis, such as an extra-abdominal desmoid tumor, tends to be large (several centimeters) and occurs most commonly in the abdominal wall, trunk, head, and neck. Superficial fibromatosis occurs in the palm of the hand and the sole of the foot. Fibromatosis tends to have an infiltrative margin on imaging. ${ }^{20}$

It is interesting to note that the radiologists who interpreted the cases did not entertain the prospective diagnosis of nodular fasciitis on the radiologic reports. This factor may have been due to lack of clinical information at the time of imaging or to the lack of familiarity with the entity. Radiologists are well aware of the imaging features of peripheral nerve sheath tumors on MRI but may be less familiar with the appearance on sonography. A peripheral nerve sheath tumor is a less likely diagnosis if a nerve is not seen in contiguity with the lesion, both entering and exiting the lesion. A peripheral nerve sheath tumor will usually show increased color Doppler flow, and a fascial tail is not associated with this entity.

In 7 cases, the preliminary pathologic diagnosis on core needle biopsy of nodular fasciitis was subsequently changed when the lesions were resected and sent for histologic analysis of the resection specimens. There is some degree of overlap between nodular fasciitis and other benign entities. However, if a correlation is made with the imaging findings discussed here, then accuracy may be increased. It is interesting to note that in none of our cases was a diagnosis of sarcoma or other malignant entity made on percutaneous core biopsy. In addition, no cases with the core biopsy result of nodular fasciitis resulted in a change of diagnosis to a malignant entity.

Although the number of cases in this study was relatively small, this experience demonstrates that core biopsy of nodular fasciitis lesions can be unreliable due to sampling errors. Gross resection or excisional biopsy is necessary to make an accurate diagnosis so that the specimen can be examined in its entirety for the characteristic findings of nodular fasciitis.

We acknowledge several limitations of this study. The first limitation is the small number of cases. Although ours is a tertiary referral center for sarcomas, and we expected our search of the radiologic and pathologic databases over a 10 -year period to yield a great number of cases, we were surprised to find only 19 potential cases, resulting in 6 cases in our final study population. Although the literature states that nodular fasciitis is common, our experience proves otherwise. The study was retrospective in nature, which was necessary as this diagnosis is infrequently made, and it would have taken decades to accumulate the number of cases necessary for a prospective study. We also reviewed the cases in consensus, in an effort to collectively characterize the imaging features of nodular fasciitis.

In conclusion, the imaging features of nodular fasciitis that we found common to all lesions are an oval or a round shape with irregular or lobular margins, a fascial tail, hypoechogenicity on sonography, and fat surrounding the lesion on MRI. When these imaging features are recognized, the diagnosis of nodular fasciitis should be entertained, and, more importantly, the possibility of an inaccurate diagnosis by core biopsy exists, which may warrant gross resection.

\section{References}

1. Konwaler BE, Keasbey L, Kaplan L. Subcutaneous pseudosarcomatous fibromatosis (fasciitis). Am J Clin Pathol 1955; 25:241-252.

2. Nikolaidis P, Gabriel HA, Lamba AR, Chan NG. Sonographic appearance of nodular fasciitis. J Ultrasound Med 2006; 25:281-285.

3. Bernstein KE, Lattes R. Nodular (pseudosarcomatous) fasciitis, a nonrecurrent lesion: clinicopathologic study of 134 cases. Cancer 1982; 49:1668-1678

4. Erickson-Johnson MR, Chou MM, Evers BR, et al. Nodular fasciitis: a novel model of transient neoplasia induced by MYH9-USP6 gene fusion. Lab Invest 201 1; 91:1427-1433.

5. KransdorfMJ. Benign soft-tissue tumors in a large referral population: distribution of specific diagnoses by age, sex, and location. AJRAmJ Roentgenol 1995; 164:395-402.

6. Hayashi $\mathrm{H}$, Nishikawa M, Watanabe R, et al. Nodular fasciitis of the breast. Breast Cancer 2007; 14:337-339.

7. Dinauer PA, Brixey CJ, Moncur JT, Fanburg-Smith JC, Murphey MD Pathologic and MR imaging features of benign fibrous soft-tissue tumors in adults. Radiographics 2007; 27:173-187.

8. Leung LY, Shu SJ, Chan AC, Chan MK, Chan CH. Nodular fasciitis: MRI appearance and literature review. Skeletal Radiol 2002; 31:9-13.

9. Grobmyer SR, Knapik JA, Foss RM, Copeland EM, Hochwald SN. Nodular fasciitis: differential considerations and current management strategies. Am Surg 2009; 75:610-614.

10. Shimizu S, Hashimoto H, Enjoji M. Nodular fasciitis: an analysis of 250 patients. Pathology 1984; 16:161-166.

11. Coyle J, White LM, Dickson B, Ferguson P, Wunder J, Naraghi A. MRI characteristics of nodular fasciitis of the musculoskeletal system. Skeletal Radiol 2013; 42:975-982.

12. GelfandJM, Mirza N, Kantor J, et al. Nodular fasciitis.Arch Dermatol 2001; 137:719-721

13. Bancroft LW, Peterson JJ, Kransdorf MJ, Nomikos GC, Murphey MD Soft tissue tumors of the lower extremities. Radiol Clin North Am 2002; 40:991-1011. 
14. Wang XL, De Schepper AM, Vanhoenacker F, et al. Nodular fasciitis: correlation of MRI findings and histopathology. Skeletal Radiol 2002; 31:155-161.

15. Yanagisawa A, Okada H. Nodular fasciitis with degeneration and regression. J Craniofac Surg 2008; 19:1167-1170.

16. Matsuzaki T, Akisue T, Kishimoto K, et al. Intra-articular nodular fasciitis of the knee: a rare cause of recurrent hemarthrosis. Rheumatol Int 2012; 32:1691-1694.

17. Harish S, Kuruvilla M, Alowami S, DeNardi F, Ghert M. Intra-articular nodular fasciitis of the shoulder: a case report and review of the literature. Skeletal Radiol 2011; 40:1383-1386.

18. Le Corroller T, Kovacs TJ, Champsaur P. Nodular fasciitis with cortical involvement. Joint Bone Spine 2009; 76:101-103.

19. Murphey MD, Smith WS, Smith SE, Kransdorf MJ, Temple HT. From the archives of the AFIP. Imaging of musculoskeletal neurogenic tumors: radiologic-pathologic correlation. Radiographics 1999; 19:1253-1280.

20. Murphey MD, Ruble CM, Tyszko SM, Zbojniewicz AM, Potter BK, Miettinen M. From the archives of the AFIP. Musculoskeletal fibromatoses: radiologic-pathologic correlation. Radiographics 2009; 29:21432173. 